\title{
NEW ORBITAL PARAMETERS AND RADIAL VELOCITY CURVE ANALYSIS OF SPECTROSCOPIC BINARY STARS
}

\author{
Kamal Ghaderi, Ali Pirkhedri, Touba Rostami, Salem Khodamoradi, and Hedayat Fatahi \\ Department of Science and Engineering, Islamic Azad University, Marivan Branch, Marivan, Iran \\ E-mail : K.Ghaderi.60@gmail.com \\ (Received December 1, 2011; Revised December 25, 2011; Accepted December 26, 2011)
}

\begin{abstract}
We use a Probabilistic Neural Network (PNN) technique to derive the orbital parameters of spectroscopic binary stars. Using measured radial velocity data of five double-lined spectroscopic binary systems (i.e., EQ Tau, V376 And, V776 Cas, V2377 Oph and EE Cet), we find the corresponding orbital and spectroscopic elements. Our numerical results are in good agreement with those obtained by other groups via more traditional methods.
\end{abstract}

Key words : Stars, binaries, eclipsing — Stars, binaries, spectroscopic

\section{INTRODUCTION}

Determining the orbital elements of binary stars allows one to obtain fundamental information, such as the mass and radius of stars; their knowledge plays an important role in the evolution of stellar objects. Through the analysis of both the light and radial velocity (hereafter $\mathrm{V}_{\mathrm{R}}$ ) curves deduced from photometric and spectroscopic observations, respectively, we can infer the complete orbital parameters. There are different methods to determine the orbit of a spectroscopic binary from its $\mathrm{V}_{\mathrm{R}}$ curve. Lehmann-Filhés (1894) introduced a geometrical technique to determine the orbital elements from the geometrical properties of the $\mathrm{V}_{\mathrm{R}}$ curve, especially its maxima and minima. The method of Lehmann-Filhés (1984) has been very useful and little, if any, longer than other methods, providing a planimeter is used. In order to improve the final solution of the orbit, their technique also provides the differential corrections to the preliminary elements, using the form of the equations obtained by the method of least squares. Russell (1902) suggested an analytical technique to develop the observed $V_{R}$ into a trigonometric series (Fourier series), and the elements are found by comparing this series with the corresponding analytical expression for the velocity. In this method the time consumed is considerably longer than that of Lehmann-Filhés (1984), and when the eccentricity is much greater than 0.4 , it becomes laborious. King (1908) proposed a graphical method which enables one to use the entire velocity curve in finding the preliminary elements, and is applicable to orbits of any eccentricity. This method depends on the velocities at equal time intervals as read from a freehand curve drawn to represent the observations. Schlesinger (1910) presented the least square method, which differ-

Corresponding Author: K. Ghaderi entially corrects the preliminary orbital elements using the equations of condition. Schlesinger's method is suitable for all the orbits except those with very small eccentricities. It has a greater accuracy and it also enables one to vary all the unknown parameters simultaneously, instead of one or two at the time. Russell (1914) developed a quick method analogous to the graphical technique of King (1908), equally applicable to orbits with all eccentricities. It has the advantage that a single diagram replaces numerous protractors which must be constructed for each separate value of the eccentricity. Within king's method, the graphical processes which require precise drawings of lines and measurements of angles will consume more time than the quick strategy of Russell (1914). Sterne (1941) described two forms of least square solutions, both of which allow for the differential corrections to be set of preliminary elements obtained by any direct method like those of Russell $(1902,1914)$. The first form is a modification of the Schlesinger's method (1910), in which the date of periastron passage is replaced by the date at which the mean longitude is zero. The first form is suitable for all the orbits except those with very small eccentricities. The second form is particularly suitable for orbits having very small eccentricities. Singh (1984) introduced an approach similar to the method of Russell (1902) for expanding the $\mathrm{V}_{\mathrm{R}}$ as a trigonometric series. This technique gives a standard set of velocity curves for a set of eccentricity, and the longitude of periastron values that can be used for the determination of preliminary elements preparatory to a least square correction. Karami and Teimoorinia (2007) introduced a new non-linear least squares velocity curve analysis technique for spectroscopic binary stars. Their method can be applied to orbits of all eccentricities and inclination angles, and the required time is considerably less than the one obtained with method of LehmannFilhés (1984). They showed the validity of their new 


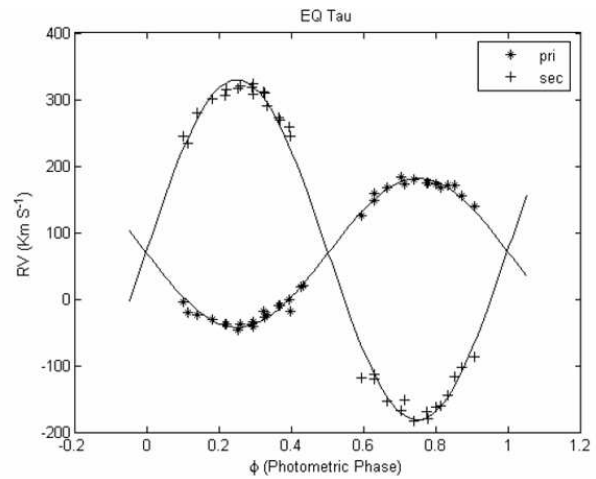

Fig. 1. - Radial velocities of the primary and secondary components of EQ Tau, plotted against the photometric phase. The observational data have been measured by Rucinski et al. (2001).

method using a wide range of different types of binaries - see Karami, Mohebi $(2007 a, b, 2009)$ and Karami et al. (2008).

So far, a large number of practical methods have been proposed. However, it is not useful to discuss their relative advantages: the method which gives the best result in one case may be unsuitable in another. For instance, for near circular orbits and small eccentricity, the $\mathrm{V}_{\mathrm{R}}$ curve approaches the simple sine curve and graphical methods are not ideal. In that case, analytical methods become useful - see Curtis (1908), Plummer (1908) and Sterne (1941). Lucy and Sweeney (1971) suggested that, because of observational errors, most of the spectroscopic binaries with eccentricities close to zero should be assigned circular orbits. Their argument is based purely statistical considerations, regardless of the orbital period, and has been subsequently challenged by other authors. For instance, Skuljan et al. (2004) emphasize that modern observations do support the idea of Lucy and Sweeney (1971), at least for the shortest-period binaries (e.g., with period less than about 10 days for late-type dwarfs). However, the situation for longer periods is not clear, and the single-lined spectroscopic binary star $\zeta \operatorname{Tr} A$ is one such examples. They point out that the orbit of $\zeta$ $\operatorname{Tr} \mathrm{A}$ was proved to be a definite ellipse, although with an extremely small eccentricity equal to 0.01442 . There is always the possibility that a third low mass unseen component could have perturbed the orbit of $\zeta \operatorname{TrA}$ to non-circularity. For the evolution of low mass members of close binary systems see Han et al. (2000), Yakut and Eggleton (2005).

Artificial Neural Networks (ANNs) have become a popular tool in almost every field of science. In recent years, ANNs have been widely used in astronomy for applications such as star/galaxy discrimination, morphological classification of galaxies, and spectral classification of stars (see Bazarghan et al. 2008, and references therein). Following Bazarghan et al. (2008), we employ Probabilistic Neural Networks (PNNs), which

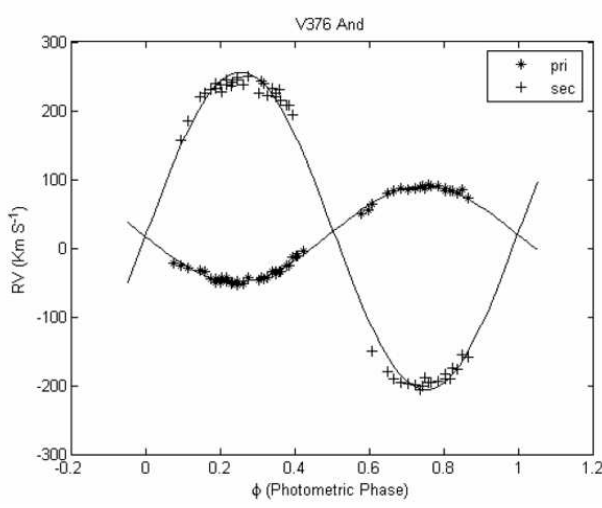

Fig. 2. - Same as Fig. 1, but for V376 And.

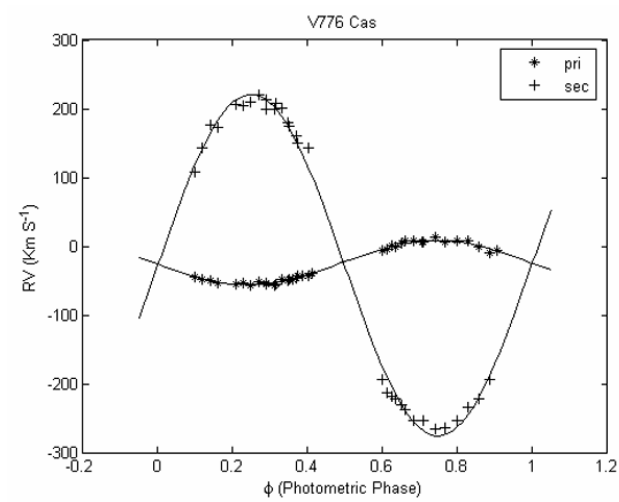

Fig. 3.- Same as Fig. 2, but for V776 Cas.

have been investigated in detail by Bazarghan et al. (2008).

PNNs are a new tool to derive the orbital parameters of spectroscopic binary stars. In this method, the time consumed is considerably inferior to that of Lehmann-Filhés, and even less than the non-linear regression method proposed by Karami \& Teimoorinia (2007). In the present paper, we use a PNN technique to find the optimum match to the four parameters of the $V_{R}$ curves of the five double-lined spectroscopic binary systems: EQ Tau, V376 And, V776 Cas, V2377 Oph, and EE Cet. Our aim is to show the validity of our new method for a wide range of binaries of different types.

In particular, EQ Tau is a close binary system. The spectral type is $\mathrm{G} 2$, with a period of $\mathrm{P}=0.341348$ days (Rucinski et al. 2001). V376 And is a contact binary of W UMa-type with spectral type A4V. The relatively long period of 0.799 days is consistent with the spectral type (Rucinski et al. 2001). V776 Cas is a contact Atype system with a very small mass ratio due to a low orbital inclination. The spectral type is F2V and the orbital period is 0.440413 days (Rucinski et al. 2001). V2377 Oph is a fairly uncomplicated W-type contact binary. The spectral type is $\mathrm{G} 0 / 1 \mathrm{~V}$ and the orbital period is 0.425401 days (Lu et al. 2001). EE Cet is a 


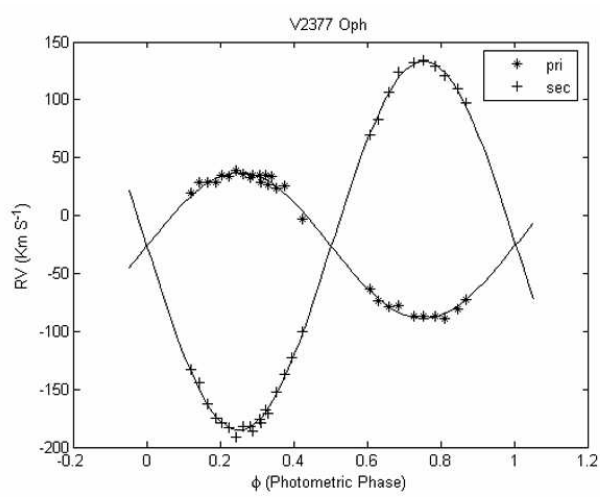

Fig. 4. - Radial velocities of the primary and secondary components of V2377 Oph, plotted against the photometric phase. The observational data have been measured by $\mathrm{Lu}$ et al. (2001).

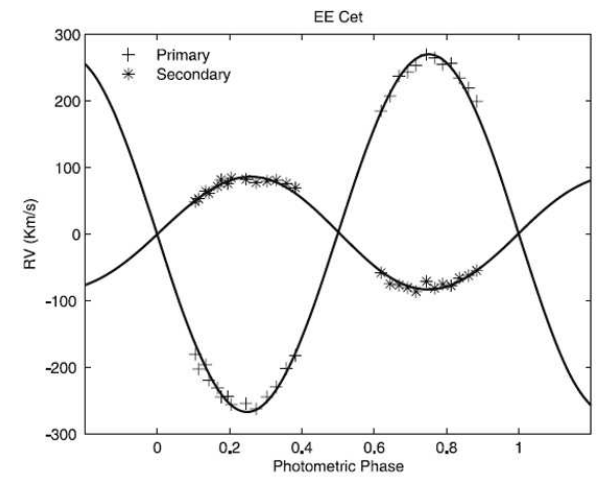

Fig. 5. - Radial velocities of the primary and secondary components of EE cet, plotted against the photometric phase. The observational data have been measured by Rucinski et al. (2002a,b).

contact double-lined spectroscopic binary with a period of $\mathrm{P}=0.339917$ days (Rucinski et al. $2002 a, b$ ).

This paper is organized as follows. In Section 2, we introduce a Probabilistic Neural Network (PNN) method to estimate the four parameters of the $V_{R}$ curve. Section 3 contains the numerical results. Conclusions are given in Section 4.

\section{PNN PARAMETER ESTIMATION}

Following Smart (1990), the $\mathrm{V}_{\mathrm{R}}$ of a star in a binary system is defined as

$$
\mathrm{V}_{\mathrm{R}}=\gamma+K[\cos (\theta+\omega)+e \cos \omega],
$$

where $\gamma$ is the $V_{R}$ of the center of mass of the system with respect to the Sun. Also $K$ is the amplitude of the $V_{R}$ of the star with respect to the center of mass of the binary. Furthermore $\theta, \omega$ and $e$ are respectively the angular polar coordinate (true anomaly), longitude of periastron and eccentricity.
Here we apply the PNN method to estimate the four orbital parameters $\gamma, K, e$ and $\omega$ of the $\mathrm{V}_{\mathrm{R}}$ curve in Eq. (1). In this work, for the identification of the observational $V_{R}$ curves, the input vector is the fitted $V_{R}$ curve of a star. The PNN is first trained to classify the $\mathrm{V}_{\mathrm{R}}$ curves corresponding to all the possible combinations of $\gamma, K, e$ and $\omega$. One can synthetically generate $\mathrm{V}_{\mathrm{R}}$ curves given by Eq. (1), for each combination of the parameters:

- $-100 \leq \gamma \leq 100$ in steps of 1 ;

- $1 \leq K \leq 300$ in steps of 1 ;

- $0 \leq e \leq 1$ in steps of 0.001 ;

- $0 \leq \omega \leq 360^{\circ}$ in steps of $5^{\circ}$.

The training procedure provides a large set of $\mathrm{k}$ pattern groups, where $\mathrm{k}$ denotes the number of different $\mathrm{V}_{\mathrm{R}}$ classes, one class for each combination of $\gamma, K, e$ and $\omega$. Since the large number of different $V_{R}$ classes causes some computational limitations, one can first start with larger step-sizes. Note that, according to Petrie (1960), one can guess $\gamma, K$ and $e$ from the $\mathrm{V}_{\mathrm{R}}$ curve. This enables one to restrict the range of parameters around their initial guesses. When the preliminary orbit is derived, one can use smaller step-sizes to obtain the final orbit. The PNN has four layers: input, pattern, summation, and output, respectively (see Fig. 5 in Bazarghan et al. 2008). When an input vector is present, the pattern layer computes distances from the input vector to the training input vectors and produces a vector whose elements indicate how close the input is to a training input. The summation layer adds these contributions for each class of inputs, to produce a vector of probabilities. Finally, a competitive transfer function on the output layer picks the maximum of these probabilities, and returns one for the current class, and zero for the others (Specht 1988, 1990). Thus, the PNN classifies the input vector into a specific k class labeled by the four parameters $\gamma, K$, $e$ and $\omega$, since that class has the maximum probability of occurrence.

\section{NUMERICAL RESULTS}

In this section we use the PNN method to derive the orbital elements for five different double-lined spectroscopic systems (i.e., EQ Tau, V376 And, V776 Cas, V2377 Oph and EE Cet). With measured $V_{\mathrm{R}}$ data for the two components of these systems (EQ Tau, V376 And, V776 Cas are data from Rucinski et al. 2001, V2377 Oph is from Lu et al. 2001, while EE Cet is taken from Rucinski et al. 2002a,b), we plot in figs. 1-5 the velocity curves as a function of their phases.

The orbital parameters obtained from the PNN for EQ Tau, V376 And, V776 Cas, V2377 Oph and EE Cet are reported in Tables 1, 3, 5, 7 and 9, respectively. The various tables show that results are in good agreement with those obtained by Rucinski et al. (2001) for EQ 
Table 1.

Orbital parameters of EQ Tau

\begin{tabular}{lll}
\hline \hline & This Paper & Rucinski et al. (2001) \\
\hline$\gamma\left[\mathrm{kms}^{-1}\right]$ & $72 \pm 1$ & $71.95(1.22)$ \\
$K_{p}\left[\mathrm{kms}^{-1}\right]$ & $112 \pm 1$ & $112.41(1.43)$ \\
$K_{s}\left[\mathrm{kms}^{-1}\right]$ & $255 \pm 1$ & $254.38(2.42)$ \\
$e$ & $0.002 \pm 0.001$ & - \\
$\omega\left[^{\circ}\right]$ & $290 \pm 5$ & - \\
\hline \hline
\end{tabular}

Table 2.

Combined spectroscopic elements of EQ Tau

\begin{tabular}{ll}
\hline \hline Parameter & This Paper $\quad$ Rucinski et al. (2001) \\
\hline$m_{p} \sin ^{3} i / M_{\odot}$ & $1.2147 \pm 0.0180-$ \\
$m_{s} \sin ^{3} i / M_{\odot}$ & $0.5335 \pm 0.0106-$ \\
$\left(m_{p}+m_{s}\right) \sin ^{3} i / M_{\odot}$ & $1.7482 \pm 0.02861 .749(55)$ \\
$a_{p} \sin i / R_{\odot}$ & $0.7553 \pm 0.0067-$ \\
$a_{s} \sin i / R_{\odot}$ & $1.7197 \pm 0.0067-$ \\
$\left(a_{p}+a_{s}\right) \sin i / R_{\odot}$ & $2.4751 \pm 0.0135-$ \\
$m_{s} / m_{p}$ & $0.4392 \pm 0.00560 .442(7)$ \\
\hline \hline
\end{tabular}

Table 3.

Orbital parameters of V376 And

\begin{tabular}{lll}
\hline \hline & This Paper & Rucinski et al. (2001) \\
\hline$\gamma\left[\mathrm{kms}^{-1}\right]$ & $23 \pm 1$ & $22.83(0.89)$ \\
$K_{p}\left[\mathrm{kms}^{-1}\right]$ & $70 \pm 1$ & $70.00(0.67)$ \\
$K_{s}\left[\mathrm{kms}^{-1}\right]$ & $230 \pm 1$ & $229.67(1.78)$ \\
$e$ & $0.004 \pm 0.001$ & - \\
$\omega\left[^{\circ}\right]$ & $275 \pm 5$ & - \\
\hline \hline
\end{tabular}

Table 4.

Combined spectroscopic elements of V376 And

\begin{tabular}{ll}
\hline \hline Parameter & This Paper $\quad$ Rucinski et al. (2001) \\
\hline$m_{p} \sin ^{3} i / M_{\odot}$ & $1.7135 \pm 0.0303-$ \\
$m_{s} \sin ^{3} i / M_{\odot}$ & $0.5215 \pm 0.0144-$ \\
$\left(m_{p}+m_{s}\right) \sin ^{3} i / M_{\odot}$ & $2.2351 \pm 0.04472 .232(55)$ \\
$a_{p} \sin i / R_{\odot}$ & $1.1050 \pm 0.0158-$ \\
$a_{s} \sin i / R_{\odot}$ & $3.6307 \pm 0.0158-$ \\
$\left(a_{p}+a_{s}\right) \sin i / R_{\odot}$ & $4.7358 \pm 0.0316-$ \\
$m_{s} / m_{p}$ & $0.3043 \pm 0.00570 .305(5)$ \\
\hline \hline
\end{tabular}

Table 5.

Orbital parameters of V776 Cas

\begin{tabular}{lll}
\hline \hline & This Paper & Rucinski et al. (2001) \\
\hline$\gamma\left[\mathrm{kms}^{-1}\right]$ & $-25 \pm 1$ & $-24.71(1.11)$ \\
$K_{p}\left[\mathrm{kms}^{-1}\right]$ & $32 \pm 1$ & $31.97(0.64)$ \\
$K_{s}\left[\mathrm{kms}^{-1}\right]$ & $245 \pm 1$ & $245.31(1.83)$ \\
$e$ & $0.001 \pm 0.001$ & - \\
$\omega\left[^{\circ}\right]$ & $285 \pm 5$ & - \\
\hline \hline
\end{tabular}

Tau, V376 And and V776 Cas, Lu et al. (2001) for V2377 Oph, and Rucinski et al. $(2002 a, b)$ for EE Cet.

Note that the Gaussian errors of the orbital parameters in Tables 1, 3, 5, 7 and 9 are the same selected steps for generating $\mathrm{V}_{\mathrm{R}}$ curves, i.e., $\Delta \gamma=1, \Delta K=$ $1, \Delta e=0.001$ and $\Delta \omega=5$. These are close to the observational errors reported in the literature. Regarding the estimated errors, following Specht (1990) the error of the decision boundaries depends on the accuracy with which the underlying Probability Density Functions (PDFs) are estimated. Parzen (1962) proved that the expected error gets smaller, as the estimate is based on a large data set. This definition of consistency is particularly important since the true distribution will be approached smoothly. Specht (1990) showed that a very large value of the smoothing parameter would cause the estimated errors to be Gaussian regardless of the true underlying distribution and the misclassification rate is stable and does not change dramatically with small changes of the smoothing parameter. The combined spectroscopic elements, including $m_{p} \sin ^{3} i$, $m_{s} \sin ^{3} i,\left(m_{p}+m_{s}\right) \sin ^{3} i,\left(a_{p}+a_{s}\right) \sin i$ and $m_{s} / m_{p}$, are calculated by substituting the estimated parameters $K, e$ and $\omega$ into Eqs. (3), (15) and (16) in Karami $\&$ Teimoorinia (2007). The results obtained for the five systems are reported in Tables 2, 4, 6, 8 and 10 they show that our results are in good agreement with those obtained by Rucinski et al. (2001) for EQ Tau, V376 And and V776 Cas, Lu et al. (2001) for V2377 Oph and Rucinski et al. $(2002 a, b)$ for EE Cet, respectively. The errors of the combined spectroscopic elements in tables 2, 4, 6, 8 and 10 are obtained by the help of orbital parameters errors - see again Eqs. (3), (15) and (16) in Karami and Teimoorinia (2007).

\section{CONCLUSIONS}

We applied a Probabilistic Neural Network (PPN) technique to derive the orbital elements of spectroscopic binary stars. PNNs are used in both regression (including parameter estimation) and classification problems. However, one can discretize a continuous regression problem to such a degree that it can be represented as a classification problem (Specht 1988, 1990), as we did in this work.

Using the measured $\mathrm{V}_{\mathrm{R}}$ from data of EQ Tau, V376 And, V776 Cas, V2377 Oph and EE Cet obtained by Rucinski et al. (2001), Lu et al. (2001) and Rucinski et al. $(2002 a, b)$, respectively, we find the orbital elements of these systems by the PNN technique. Our numerical results show that the orbital and spectroscopic parameters are in good agreement with those obtained by other groups using more traditional methods.

Our technique is applicable to orbits of all eccentricities and inclination angles. In our method, the time spent to recover the various parameters is considerably inferior to that of Lehmann-Filhés. It is also more accurate as the orbital elements are deduced from all the 
Table 6

Combined spectroscopic elements of V776 Cas

\begin{tabular}{ll}
\hline \hline Parameter & This Paper $\quad$ Rucinski et al. (2001) \\
\hline$m_{p} \sin ^{3} i / M_{\odot}$ & $0.8578 \pm 0.0159-$ \\
$m_{s} \sin ^{3} i / M_{\odot}$ & $0.1120 \pm 0.0051-$ \\
$\left(m_{p}+m_{s}\right) \sin ^{3} i / M_{\odot}$ & $0.9698 \pm 0.02100 .975(26)$ \\
$a_{p} \sin i / R_{\odot}$ & $0.2784 \pm 0.0087-$ \\
$a_{s} \sin i / R_{\odot}$ & $2.1318 \pm 0.0087-$ \\
$\left(a_{p}+a_{s}\right) \sin i / R_{\odot}$ & $2.4103 \pm 0.0174-$ \\
$m_{s} / m_{p}$ & $0.1306 \pm 0.00460 .130(4)$ \\
\hline \hline
\end{tabular}

Table 7.

Orbital parameters of V2377 Oph

\begin{tabular}{lll}
\hline \hline & This Paper & Lu et al. (2001) \\
\hline$\gamma\left[\mathrm{kms}^{-1}\right]$ & $-25 \pm 1$ & $-25.79(3.80)$ \\
$K_{p}\left[\mathrm{kms}^{-1}\right]$ & $63 \pm 1$ & $62.99(0.62)$ \\
$K_{s}\left[\mathrm{kms}^{-1}\right]$ & $160 \pm 1$ & $159.64(0.70)$ \\
$e$ & $0.004 \pm 0.001$ & - \\
$\omega\left[^{\circ}\right]$ & $45 \pm 5$ & - \\
\hline \hline
\end{tabular}

Table 8.

Combined spectroscopic elements of V2377 Oph

\begin{tabular}{ll}
\hline \hline Parameter & This Paper $\quad$ Lu et al. (2001) \\
\hline$m_{p} \sin ^{3} i / M_{\odot}$ & $0.3507 \pm 0.0085-$ \\
$m_{s} \sin ^{3} i / M_{\odot}$ & $0.1381 \pm 0.0047-$ \\
$\left(m_{p}+m_{s}\right) \sin ^{3} i / M_{\odot}$ & $0.4888 \pm 0.01320 .487(9)$ \\
$a_{p} \sin i / R_{\odot}$ & $0.5295 \pm 0.0084-$ \\
$a_{s} \sin i / R_{\odot}$ & $1.3447 \pm 0.0084-$ \\
$\left(a_{p}+a_{s}\right) \sin i / R_{\odot}$ & $1.8742 \pm 0.0168-$ \\
$m_{s} / m_{p}$ & $0.3937 \pm 0.00870 .395(12)$ \\
\hline \hline
\end{tabular}

Table 9.

Orbital parameters of EE Cet

\begin{tabular}{lll}
\hline \hline & This Paper & Rucinski et al. (2002a,b) \\
\hline$\gamma\left[\mathrm{kms}^{-1}\right]$ & $2 \pm 1$ & $1.6(0.93)$ \\
$K_{p}\left[\mathrm{kms}^{-1}\right]$ & $267 \pm 1$ & $266.92(1.54)$ \\
$K_{s}\left[\mathrm{kms}^{-1}\right]$ & $84 \pm 1$ & $84.05(1.24)$ \\
$e$ & $0.001 \pm 0.001$ & - \\
$\omega\left[^{\circ}\right]$ & $305 \pm 5$ & - \\
\hline \hline
\end{tabular}

points of the velocity curve instead of just four as in the method of Lehmann-Filhés. The present method enables one to vary all the unknown parameters $\gamma, K$, $e$ and $\omega$ simultaneously instead of one or two of them at the time. It is possible to make adjustments in the elements before the final result is obtained. There are some cases for which the geometrical methods are inapplicable, and in these situations the present technique may be useful. For example, when observations are in-
Table 10

Combined spectroscopic elements of EE Cet

\begin{tabular}{ll}
\hline \hline Parameter & This Paper $\quad$ Rucinski et al. (2002a,b) \\
\hline$m_{p} \sin ^{3} i / M_{\odot}$ & $0.3645 \pm 0.0085-$ \\
$m_{s} \sin ^{3} i / M_{\odot}$ & $1.1585 \pm 0.0175-$ \\
$\left(m_{p}+m_{s}\right) \sin ^{3} i / M_{\odot}$ & $1.5229 \pm 0.02601 .706(41)$ \\
$a_{p} \sin i / R_{\odot}$ & $1.7931 \pm 0.0067-$ \\
$a_{s} \sin i / R_{\odot}$ & $0.5641 \pm 0.0067-$ \\
$\left(a_{p}+a_{s}\right) \sin i / R_{\odot}$ & $2.3572 \pm 0.0134-$ \\
$m_{p} / m_{s}$ & $0.3146 \pm 0.00490 .315(5)$ \\
\hline
\end{tabular}

complete because certain phases could not have been observed, or when a star is surrounded by two dark companions with commensurable periods. In the latter situation, the resultant velocity curve may have several unequal maxima, causing the geometrical methods to fail.

\section{REFERENCES}

Bazarghan, M., Safari, H., Innes, D. E., Karami, E., \& Solanki, S. K. 2008, A Nanoflare Model for Active Region Radiance: Application of Artificial Neural Networks, A\&A, 492, L13

Curtis, H. D. 1908, Methods of Determining the Orbits of Spectroscopic Binaries, PASP, 20, 133

Han, Z., Tout, C. A., \& Eggleton, P. P. 2000, Low and Intermediate-Mass Close Binary Evolution and the Initial - Final Mass Relation, MNRAS, 319, 215

Karami, K., \& Mohebi, R. 2007a, Velocity Curve Analysis of Spectroscopic Binary Stars AI Phe, GM Dra, HD 93917 and V502 Oph by Nonlinear Regression, ChJAA, 7, 558

Karami, K., \& Mohebi, R. 2007b, Velocity Curve Analysis of Spectroscopic Binary Stars PV Pup, HD141929, EE Cet and V921 Her by Nonlinear Regression, JApA, 28, 217

Karami, K., \& Teimoorinia, H. 2007, Velocity Curve Analysis of the Spectroscopic Binary Stars by the Non-linear Least Squares, Ap\&SS, 311, 435

Karami, K., Mohebi, R., \& Soltanzadeh, M. M. 2008, Application of a New Non-Linear Least Squares Velocity Curve Analysis Technique for Spectroscopic Binary Stars, Ap\&SS, 318, 69

Karami, K., \& Mohebi, R. 2009, Velocity Curve Studies of Spectroscopic Binary Stars V380 Cygni, V401 Cyg, V523 Cas, V373 Cas, and V2388 Oph, JApA, 30,153

King, W. F. 1908, Determination of the Orbits of Spectroscopic Binaries, ApJ, 27, 125

Lehmann-Filhés, R. 1894, Ueber Die Bestimung Einer Doppelsternbahn Aus Spectroskopischen Messungen Der Im Visionsradius Liegenden Geschwindigkeits componente, AN, 136, 17 
Lu, W., Rucinski, S. M., \& Ogloza, W. 2001, Radial Velocity Studies of Close Binary Stars. IV, AJ, 122, 402

Lucy, L. B., \& Sweeney, M. A. 1971, Spectroscopic Binaries with Circular Orbits, AJ, 76, 544.

Parzen, E. 1962, On Estimation of a Probability Density Function and Mode. Annals of Mathematical Statistics, 33, 1065

Petrie, R. M. 1960, Astronomical Techniques, University of Chicago Press

Plummer, H. C. 1908, Notes on The Determinations of The Orbits of Spectroscopic Binaries, ApJ, 28, 212

Rucinski, S. M., Lu, W., Mochnaki, S. W., Ogloza, W., \& Stachowski, G. 2001, Radial Velocity Studies of Close Binary Stars. V, AJ, 122, 1974

Rucinski, S. M., Capobianco, C. C., Lu, W., Mochnaki, S. W., Blake, R. M., Thomson, J. R., Ogloza W., \& Stachowski, W. 2002a, Radial Velocity Studies of Close Binary Stars. VI, AJ, 124, 1738

Rucinski, S. M., Capobianco, C. C., Lu, W., Mochnaki S. W., Blake, R. M., Thomson, J. R., Ogloza, W., \& Stachowski, W. 2002b, Radial Velocity Studies of Close Binary Stars. VI, preprint (astro-ph/0201213)

Russell, H. N. 1902, An Improved Method of Calculating the Orbit of a Spectroscopic Binary, ApJ, 15, 252

Russell, H. N. 1914, A Short Method For Determining the Orbit of a Spectroscopic Binary, ApJ, 40, 282

Schlesinger, F. 1910, The Algol-Variable $\delta$ Librae, PALLO, 1, 123

Singh, M. 1984, The Determination of a Spectroscopic Binary Orbit, Ap\&SS, 100, 13

Skuljan, J., Ramm, D. J., \& Hearnshaw, J. B. 2004, Accurate Orbital Parameters For The Bright Southern Spectroscopic Binary $\zeta$ Trianguli Australis An Interesting Case of a Near-Circular Orbit, MNRAS, 352,975

Smart, W. M. 1990, Textbook on Spherical Astronomy, Sixth Ed., Cambridge Univ. Press, p. 360

Specht, D. F. 1988, Probabilistic Neural Networks for Classification, Mapping, or Associative Memory, In Proceedings of the IEEE International Conference on Neural Networks, San Diego, 24-27 July, p. 525

Specht, D. F. 1990, Probabilistic Neural Networks, Neural Networks, 3, 109

Sterne, T. E. 1941, Notes on Binary Stars. V. The Determination by Least-Squares of the Elements of Spectroscopic Binaries, PNAS, 27, 175

Yakut, K., \& Eggleton, P. P. 2005, Evolution of Close Binary Systems, ApJ, 629, 1055 